\title{
Zeros of the constant term in the Chowla-Selberg formula
}

by

\section{HASEO KI (Seoul)}

1. Introduction. We consider the Eisenstein series

$$
E_{0}(z ; s)=\frac{1}{2} \sum_{(m, n) \neq(0,0)} \frac{y^{s}}{|m z+n|^{2 s}} \quad(\operatorname{Re}(s)>1)
$$

where $z=x+y i, x \in \mathbb{R}, y>0$. This series has the functional equation

$$
\pi^{-s} \Gamma(s) E_{0}(z ; s)=\pi^{-1+s} \Gamma(1-s) E_{0}(z ; 1-s),
$$

where $\Gamma(s)$ is the gamma function. Also $E_{0}(z ; s)$ can be analytically continued to the complex plane except for the simple pole at $s=1$. In the present context, the Chowla-Selberg formula asserts that

$$
\begin{aligned}
E_{0}(z ; s)= & \zeta(2 s) y^{s}+\sqrt{\pi} \frac{\Gamma(s-1 / 2)}{\Gamma(s)} \zeta(2 s-1) y^{1-s} \\
& +4 \pi^{s} \sqrt{y} \sum_{n=1}^{\infty} n^{1 / 2-s} \sum_{d \mid n} d^{2 s-1} \frac{K_{s-1 / 2}(2 \pi n y)}{\Gamma(s)} \cos (2 \pi n x)
\end{aligned}
$$

where " $d \mid n$ " means " $d$ divides $n$ ", $\zeta(s)$ is the Riemann zeta function, and $K_{z}(a)$ is the $K$-Bessel function. For these facts see [3].

We denote the constant term in the Chowla-Selberg formula by

$$
C(z ; s)=\zeta(2 s) y^{s}+\sqrt{\pi} \frac{\Gamma(s-1 / 2)}{\Gamma(s)} \zeta(2 s-1) y^{1-s} .
$$

Using the functional equation of either $\zeta(s)$ or $E_{0}(z ; s)$, we see that

$$
\pi^{-s} \Gamma(s) C(z ; s)=\pi^{-1+s} \Gamma(1-s) C(z ; 1-s) .
$$

One also sees that $C(z ; s)$ is analytic for all $s$ except for a simple pole of residue $\pi / 2$ at $s=1$. Moreover, $C(z ; s)$ is real for $s \in \mathbb{R}-\{1\}$, positive for

2000 Mathematics Subject Classification: Primary 11M36.

This work was supported by grant No. R01-2005-000-10339-0 from the Basic Research Program of the Korea Science \& Engineering Foundation. 
$s>1,-1 / 2$ at $s=0$, zero of order 1 at $s=-1,-2,-3, \ldots$, and otherwise nonvanishing on $(-\infty, 0]$.

In this note we are interested in the complex zeros of $C(z ; s)$. Hejhal [5, Proposition 5.3] used the Maass-Selberg formula to prove that, for any $y \geq 1$, all complex zeros of $C(z ; s)$ are on $\operatorname{Re}(s)=1 / 2$. We shall supply a different proof of this result. In [5, Proposition 5.3] and [6, Theorem 2] it is proved that all but finitely many complex zeros of $C(z ; s)$ are simple for $y \geq 1$. One wonders: is it true that all complex zeros of this function are simple? We show that the answer is "yes".

TheOREM. For any $y \geq 1$ all complex zeros of $C(z ; s)$ are simple and lie on $\operatorname{Re}(s)=1 / 2$.

Concerning this theorem, we refer to [1, pp. 64-73] for some related ideas. The results in that paper are quite interesting.

It should be noted that the author [6, Corollary 1] has shown that for any $y \geq 1$ and any $N=1,2,3, \ldots$, all but finitely many complex zeros of any $N$ th partial sum in the Chowla-Selberg formula are simple and on $\operatorname{Re}(s)=1 / 2$. Since $E_{0}(i ; s)=2 \zeta(s) L\left(s, \chi_{-4}\right)$, the Riemann hypothesis would follow if, for infinitely many $N$, one was somehow able to remove the "but finitely many" clause in this corollary in the (very special) case where $z=i$.

We divide the proof of our theorem into two parts. In proving that all complex zeros of the function are on $\operatorname{Re}(s)=1 / 2$, we apply a variant of Hermite-Biehler theorem. The author used this argument to justify the second part of Theorem B in [7]. We will determine the behavior of the argument of $C(z ; s)$ which confirms the second part of our theorem.

2. Proof of the Theorem. To avoid notational confusion, it is best to change our notation slightly and speak of $C(Z ; s)$ with $Z=X+i Y$. (The variable $z$ can then be used for other purposes.) Using the functional equation for the Riemann zeta function [9, Chapter II], we see that

Set

$$
C(Z ; s)=\zeta(2 s) Y^{s}+\pi^{2 s-1} \frac{\Gamma(1-s)}{\Gamma(s)} \zeta(2-2 s) Y^{1-s} .
$$

$$
f(s)=2(s-1 / 2) s(s-1) \pi^{-s} \Gamma(s) C(Z ; s) .
$$

One readily checks that $f(s)$ is an entire function satisfying

$$
f(s)=-f(1-s)
$$

and that

$$
f(s)=(s-1) \xi(2 s) Y^{s}+s \xi(2-2 s) Y^{1-s},
$$

where

$$
\xi(s)=\frac{1}{2} s(s-1) \pi^{-s / 2} \Gamma(s / 2) \zeta(s)
$$

by $[9$, eq. (2.1.12)]. 
We need the following basic results.

Lemma 2.1. For the Riemann $\Xi$-function we have the following:

(1) $\Xi(z)=\xi\left(\frac{1}{2}+i z\right)=-\frac{1}{2}\left(z^{2}+\frac{1}{4}\right) \pi^{-1 / 4-i z / 2} \Gamma\left(\frac{1}{4}+i \frac{z}{2}\right) \zeta\left(\frac{1}{2}+i z\right)$;

$$
\Xi(z)=\Xi(0) \prod_{k=1}^{\infty}\left(1-\frac{z^{2}}{a_{k}^{2}}\right)
$$

where $a_{1}, a_{2}, a_{3}, \ldots$ enumerate all the zeros of $\Xi(z)$ in the right half-plane such that $0<\operatorname{Re}\left(a_{1}\right) \leq \operatorname{Re}\left(a_{2}\right) \leq \operatorname{Re}\left(a_{3}\right) \leq \cdots$ and $-1 / 2<\operatorname{Im}\left(a_{k}\right)<1 / 2$ for any $k=1,2,3, \ldots$;

$$
\Xi(z)=\int_{-\infty}^{\infty} \Phi(t) e^{i z t} d t
$$

where

$$
\Phi(t)=2 \sum_{n=1}^{\infty}\left(2 n^{4} \pi^{2} e^{9 t / 2}-3 n^{2} \pi e^{5 t / 2}\right) e^{-n^{2} \pi e^{2 t}} .
$$

Here $\Phi(t)=\Phi(-t)$ and $\Phi(t)>0$ for any $t \in \mathbb{R}$. Thus $\Xi(z)=\Xi(-z)$ and $\overline{\Xi(z)}=\Xi(\bar{z})$ for any complex number $z$.

For Lemma 2.1 we refer to [9, pp. 16, 30, 44, and 255].

2.1. All complex zeros of $f(s)$ are on $\operatorname{Re}(s)=1 / 2$. We put $Z=X+i Y$ in $C$ and then consider

$$
F(z)=\frac{f(1 / 2+i z)}{i \sqrt{Y}} .
$$

Using Lemma 2.1(1), we obtain

$$
F(z)=(z+i / 2) \Xi(2 z-i / 2) Y^{i z}+(z-i / 2) \Xi(2 z+i / 2) Y^{-i z} .
$$

Clearly $F(z)$ is entire and $F(-z)=-F(z)$. Moreover, $F(z)=\overline{F(\bar{z})}$.

It suffices to show that any zero $\alpha$ of $F(z)$ is either real or purely imaginary. We need the following lemma.

Lemma 2.2. Let $U(z)$ and $V(z)$ be real polynomials. Assume that $U \not \equiv 0$ and that $W(z)=U(z)+i V(z)$ has exactly $n$ zeros (counted with multiplicity) in the lower half-plane. Then $U(z)$ can have at most $n$ pairs of conjugate complex zeros (again counted with multiplicity). Similarly for the upper halfplane.

Proof. See [2, p. 215].

For each $n=1,2,3, \ldots$, we define

$$
W_{n}(z)=\Xi(0)\left(z+\frac{i}{2}\right)\left(1+\frac{i z \log Y}{n}\right)^{n} \prod_{k=1}^{n}\left(1-\frac{(2 z-i / 2)^{2}}{a_{k}^{2}}\right) .
$$


By Lemma 2.1(2) and the fact that $\log Y \geq 0$ we observe that for any $n=1,2,3, \ldots, W_{n}(z)$ has only one zero in the lower half-plane. Thus Lemma 2.2 implies that for any $n=1,2,3, \ldots, W_{n}(z)+\overline{W_{n}(\bar{z})}$ has at most one pair of conjugate complex roots. We note that $W_{n}(z)$ converges uniformly to

$$
\Xi(0)\left(z+\frac{i}{2}\right) y^{i z} \prod_{k=1}^{\infty}\left(1-\frac{(2 z-i / 2)^{2}}{a_{k}^{2}}\right)
$$

on any compact set in the complex plane. Thus $W_{n}(z)+\overline{W_{n}(\bar{z})}$ converges uniformly to $F(z)$ on any compact set in the complex plane. Hence $F(z)$ has at most one pair of conjugate complex roots. Suppose that $F\left(z_{0}\right)=0$ and, say, $\operatorname{Im}\left(z_{0}\right)>0$. Since $F$ is odd and $F(z)=\overline{F(\bar{z})}$, we see that $F$ also vanishes at $-\bar{z}_{0}$. It follows that $z_{0}=-\bar{z}_{0}$, i.e. $z_{0}$ is purely imaginary. We conclude that any zero $\alpha$ of $F(z)$ is either real or purely imaginary, and the first part of the Theorem follows.

Our proof shows that $F(z)$ has at most two complex zeros. These zeros are confined to the open interval $(-i / 2, i / 2)$ since $C(Z ; s)$ is positive for $s>1$ and has a simple pole of residue $\pi / 2$ as $s \rightarrow 1$.

Remark. For the exceptional real zeros of $C(Z ; s)$, we refer to [8, Theorem 3].

2.2. All complex zeros of $f(s)$ are simple. In the previous section, we found that all complex zeros of $f(s)$ are on $\operatorname{Re}(s)=1 / 2$. In this section, we use a more careful analysis to show that these zeros are simple.

We set

$$
\theta(t)=\arg \left(2 i t(1 / 2+i t)(-1 / 2+i t) \pi^{-1 / 2-i t} \Gamma(1 / 2+i t) \zeta(1+2 i t)\right)
$$

for $t \in \mathbb{R}$. One readily checks that

$$
f(1 / 2+i t)=0 \quad \text { precisely when } \theta(t)+t \log Y \equiv 0 \bmod \pi .
$$

For orientation purposes, it is also helpful to note that

$$
\theta^{\prime}(t)=-\log \pi+\operatorname{Re} \frac{\Gamma^{\prime}}{\Gamma}\left(\frac{1}{2}+i t\right)+2 \operatorname{Re} \frac{\zeta^{\prime}}{\zeta}(1+2 i t) .
$$

Using the standard partial fraction expansion of $\zeta^{\prime}(s) / \zeta(s)$ as in $[9$, eq. (2.12.7)], one then gets

$$
\theta^{\prime}(t)=2 b-\log \pi-\frac{2}{1+4 t^{2}}+2 \operatorname{Re} \sum_{\varrho}\left(\frac{1}{1+2 i t-\varrho}+\frac{1}{\varrho}\right)
$$

where $b=\log (2 \pi)-1-\frac{1}{2} \gamma$ and $\zeta(\varrho)=0,0<\operatorname{Re}(\varrho)<1$. Since

$$
\operatorname{Re} \frac{\Gamma^{\prime}}{\Gamma}\left(\frac{1}{2}+i t\right)=\log t+O\left(\frac{1}{t}\right), \quad \frac{\zeta^{\prime}}{\zeta}(1+2 i t)=O\left(\frac{\log t}{\log \log t}\right)
$$


(cf. [4, p. 47, (7)] and [9, eq. (5.17.4)]), the function $\theta(t)$ is increasing for large $t$; in fact,

$$
\theta^{\prime}(t) \sim \log t
$$

The mean spacing of the zeros of $f$ will thus be $\pi / \log t$ (in the limit $t \rightarrow \infty$ ). Cf. $[5$, p. 88 (middle)].

LEMMA 2.3. We have

(1) $\theta(0)=\pi$

(2) $\theta(t)>\pi / 2$ for $t>0$,

(3) $\theta(t)$ is a convex function in $(0,7)$,

(4) $\theta(t)$ increases in $[7, \infty)$.

Proof. Using Lemma 2.1(1), we have

$$
\theta(t)=\arg ((-1 / 2+i t) \Xi(2 t-i / 2)) .
$$

By $[9$, p. 30], one has $\Xi(-i / 2)=1 / 2$. We thus get $\theta(0)=\pi$, and (1) follows.

Since $\operatorname{Im}\left(a_{k}\right)>-1 / 2$ with $\Xi\left(a_{k}\right)=0(k=1,2,3, \ldots)$, we see that $\arg (\Xi(2 t-i / 2))>0$ for $t>0$. Thus we obtain

$$
\theta(t)>\arg (-1 / 2+i t)=\frac{\pi}{2}+\tan ^{-1} \frac{1}{2 t}>\frac{\pi}{2}
$$

which is $(2)$.

For the first three zeros $1 / 2+i a_{1}, 1 / 2+i a_{2}, 1 / 2+i a_{3}$ of $\zeta(s)$ in the upper half-plane, one knows that

$$
14.1<a_{1}<14.2, \quad 21<a_{2}<21.1, \quad 25<a_{3}<25.1 .
$$

For $\zeta(\varrho)=0$, we write $\varrho=\beta+i \gamma$. We note that $\zeta(\beta-i \gamma)=0$ and $0<\beta<1$. Using (2.1), we thus obtain

$$
\begin{aligned}
\theta^{\prime \prime}(t)= & \frac{16 t}{\left(1+4 t^{2}\right)^{2}} \\
& +8 \sum_{\gamma>0}\left(\frac{(1-\beta)(\gamma-2 t)}{\left[(1-\beta)^{2}+(2 t-\gamma)^{2}\right]^{2}}+\frac{(1-\beta)(-\gamma-2 t)}{\left[(1-\beta)^{2}+(2 t+\gamma)^{2}\right]^{2}}\right) .
\end{aligned}
$$

Since $0<\beta<1$ and $\gamma>14.1$, each term in the summation of (2.2) is greater than

$$
\frac{4 t(1-\beta)^{3}\left[-(1-\beta)^{2}+2\left(\gamma^{2}-4 t^{2}\right)\right]}{\left[(1-\beta)^{2}+(2 t-\gamma)^{2}\right]^{2}\left[(1-\beta)^{2}+(2 t+\gamma)^{2}\right]^{2}}>0
$$

for $t \in(0,7)$. Thus $\theta^{\prime \prime}(t)>0$ in $(0,7)$, which implies $(3)$.

We now prove (4). For this purpose, we need

FACT 2.4. Let $a>0$ and $x_{2}>x_{1}$. Then

$$
\begin{gathered}
\arg \left(x_{2}-i a\right)>\arg \left(x_{1}-i a\right), \\
\arg \left(-1 / 2+i x_{2}\right)-\arg \left(-1 / 2+i x_{1}\right)>-\frac{x_{2}-x_{1}}{2 x_{1} x_{2}} \quad\left(x_{1}>0\right),
\end{gathered}
$$




$$
\arg \left(1-\frac{2 x_{2}-i / 2}{a}\right)-\arg \left(1-\frac{2 x_{1}-i / 2}{a}\right)>\frac{1}{1+\kappa^{-2}} \frac{x_{2}-x_{1}}{\left(2 x_{1}-a\right)\left(2 x_{2}-a\right)} .
$$

In the last inequality, $\kappa=\min \left\{2\left|2 x_{1}-a\right|, 2\left|2 x_{2}-a\right|\right\}$ and we assume $2 x_{1}>a$ or $2 x_{2}<a$.

Proof. The first two inequalities are easy. For the last one, it suffices to show it for $2 x_{1}>a$, because the other case follows by the same method. We have

$$
\begin{aligned}
& \arg \left(1-\frac{2 x_{2}-i / 2}{a}\right)-\arg \left(1-\frac{2 x_{1}-i / 2}{a}\right) \\
& =\tan ^{-1} \frac{1}{2\left(2 x_{1}-a\right)}-\tan ^{-1} \frac{1}{2\left(2 x_{2}-a\right)} \\
& =\int_{\frac{1}{2\left(2 x_{2}-a\right)}}^{\frac{1}{2\left(2 x_{1}-a\right)}} \frac{1}{1+x^{2}} d x>\frac{1}{1+\kappa^{-2}} \frac{x_{2}-x_{1}}{\left(2 x_{1}-a\right)\left(2 x_{2}-a\right)}
\end{aligned}
$$

Let $t_{1}$ and $t_{2}$ be such that $13 \leq t_{1}<t_{2}$. Using Lemma 2.1(2), Fact 2.4 and $2\left(2 t_{1}-a_{k}\right)>1.8$ for $k=1,2,3$, we have

$$
\begin{aligned}
\theta\left(t_{2}\right)-\theta\left(t_{1}\right)= & \arg \left(-1 / 2+i t_{2}\right)-\arg \left(-1 / 2+i t_{1}\right) \\
& +\sum_{k=1}^{\infty} \arg \left(1-\frac{\left(2 t_{2}-i / 2\right)^{2}}{a_{k}^{2}}\right)-\arg \left(1-\frac{\left(2 t_{2}-i / 2\right)^{2}}{a_{k}^{2}}\right) \\
> & -\frac{t_{2}-t_{1}}{2 t_{1} t_{2}}+\sum_{k=1}^{3} \arg \left(1-\frac{2 t_{2}-i / 2}{a_{k}}\right)-\arg \left(1-\frac{2 t_{1}-i / 2}{a_{k}}\right) \\
> & -\frac{t_{2}-t_{1}}{2 t_{1} t_{2}}+\frac{1}{1+\left(\frac{1}{1.8}\right)^{2}} \sum_{k=1}^{3} \frac{t_{2}-t_{1}}{\left(2 t_{1}-a_{k}\right)\left(2 t_{2}-a_{k}\right)} \\
> & -\frac{t_{2}-t_{1}}{2 t_{1} t_{2}}+\frac{1}{1+\left(\frac{1}{1.8}\right)^{2}} \frac{3}{4} \frac{t_{2}-t_{1}}{t_{1} t_{2}}>0 .
\end{aligned}
$$

Let $10 \leq t_{1}<t_{2} \leq 13$. Since $5 \leq 2 t_{1}-a_{1}, 2 t_{1}-a_{1}<t_{1}$ and $2 t_{2}-a_{1}<t_{2}$, we similarly have

$$
\begin{aligned}
\theta\left(t_{2}\right)-\theta\left(t_{1}\right) & >-\frac{1}{2} \frac{t_{2}-t_{1}}{t_{1} t_{2}}+\arg \left(1-\frac{2 t_{2}-i / 2}{a_{1}}\right)-\arg \left(1-\frac{2 t_{1}-i / 2}{a_{1}}\right) \\
& >-\frac{1}{2} \frac{t_{2}-t_{1}}{t_{1} t_{2}}+\frac{1}{1+\left(\frac{1}{10}\right)^{2}} \frac{t_{2}-t_{1}}{\left(2 t_{1}-a_{1}\right)\left(t_{2}-a_{1}\right)}>0 .
\end{aligned}
$$

Let $7 \leq t_{1}<t_{2} \leq 10$. Then it is not hard to see that

$$
\frac{8}{5}>\left(\frac{a_{2}}{t_{1}}-2\right)\left(\frac{a_{2}}{t_{2}}-2\right) \text { or } \frac{1}{1+\left(\frac{1}{2}\right)^{2}} \frac{1}{\left(2 t_{1}-a_{2}\right)\left(2 t_{2}-a_{2}\right)}>\frac{1}{2} \frac{1}{t_{1} t_{2}} \text {. }
$$


Similarly, we obtain

$$
\theta\left(t_{2}\right)-\theta\left(t_{1}\right)>-\frac{1}{2} \frac{t_{2}-t_{1}}{t_{1} t_{2}}+\frac{1}{1+\left(\frac{1}{2}\right)^{2}} \frac{t_{2}-t_{1}}{\left(2 t_{1}-a_{2}\right)\left(2 t_{2}-a_{2}\right)}>0 .
$$

Thus, $\theta(t)$ is increasing in $[7, \infty)$, which completes the proof of Lemma 2.3.

By Lemma 2.3, either $\theta(t)$ is increasing in $(0, \infty)$ or there exists $t_{0} \in(0,7)$ such that $\theta(t)$ decreases in $\left(0, t_{0}\right)$ and $\theta(t)$ increases in $\left(t_{0}, \infty\right)$.

Suppose that the first case holds. Since $\log Y \geq 0$, the function $\theta(t)+$ $t \log Y$ increases in $(0, \infty)$. Thus all complex zeros of $f(s)$ are simple in $\operatorname{Im}(s)>0$.

For the second case, the proof is as follows. Similarly, all zeros of $f(s)$ in $\operatorname{Im}(s) \geq t_{0}$ are simple. By Lemma 2.3(3) it is easy to see that $\theta(t)+t \log Y$ is a convex function in $(0,7)$. By convexity and since $\theta(t)+t \log Y=\pi$ at $t=0$ and $\theta(t)+t \log Y>\pi / 2$ for $t \in(0,7)$, there exists no $t^{*} \in(0,7)$ such that $\theta(t)+t \log Y$ has a local minimum at $t^{*}$ and $\theta\left(t^{*}\right)+t^{*} \log Y=m \pi, m \geq 1$. Hence all zeros of $f(s)$ in $0<\operatorname{Im}(s) \leq t_{0}$ are simple. For the second case, we also conclude that all complex zeros of $f(s)$ are simple in $\operatorname{Im}(s)>0$.

Thus the second part of the Theorem follows.

Remark. Let $0<Y<1$. Then on $\operatorname{Re}(s)=1 / 2$ the assertion of our theorem may not be valid. But it is known (see [6, Theorem 2]) that for any $\delta>0$ all but finitely many zeros of $f(s)$ in $\{s:|\operatorname{Re}(s)-1 / 2|<\delta\}$ are simple and lie on $\operatorname{Re}(s)=1 / 2$.

Acknowledgments. The author deeply thanks the referee for his or her many valuable suggestions and comments on the paper. The author also thanks Professor Masahiko Taniguchi for his hospitality while the author was visiting the department of mathematics at Kyoto University via a JSPS Fellowship.

\section{References}

[1] C. Bays, K. Ford, R. H. Hudson, and M. Rubinstein, Zeros of Dirichlet L-functions near the real axis and Chebyshev's bias, J. Number Theory 87 (2001), 54-76.

[2] N. G. de Bruijn, The roots of trigonometric integrals, Duke Math. J. 17 (1950), 197-226.

[3] S. Chowla and A. Selberg, On Epstein's zeta-function, J. Reine Angew. Math. 227 (1967), 86-110.

[4] A. Erdélyi, Higher Transcendental Functions, Vol. 1, McGraw-Hill, 1953.

[5] D. A. Hejhal, On a result of G. Pólya concerning the Riemann $\xi$-function, J. Anal. Math. 55 (1990), 59-95.

[6] H. Ki, All but finitely many nontrivial zeros of the approximations of the Epstein zeta function are simple and on the critical line, Proc. London Math. Soc. 90 (2005), 321-344. 
[7] H. Ki, On a theorem of Levinson, J. Number Theory 107 (2004), 287-297.

[8] J. C. Lagarias and M. Suzuki, The Riemann hypothesis for certain integrals of Eisenstein series, ibid. 118 (2006), 98-122.

[9] E. C. Titchmarsh, The Theory of the Riemann Zeta-Function, 2nd ed., revised by D. R. Heath-Brown, Oxford Univ. Press, Oxford, 1986.

Department of Mathematics

Yonsei University

Seoul 120-749, Korea

E-mail: haseo@yonsei.ac.kr

Received on 22.12.2004

and in revised form on 17.4.2006 